\title{
Benign clinical and epidemiological outcomes-associated factors of COVID-19 from a solved epidemic with a low case fatality rate
}

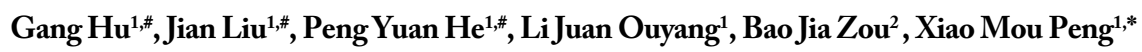 \\ ${ }^{1}$ Center of Infectious Diseases, the Fifth Affiliated Hospital, Sun Yat-sen University, China \\ ${ }^{2}$ Department of Hepatobiliary Surgery, the Fifth Affiliated Hospital, Sun Yat-sen University, China \\ \#Contributed equally to this work
}

\begin{abstract}
Background: The mortality and disease severity predictors of COVID-19 have been investigated in many studies. However, they are based on early or partial datasets from high epidemic areas. Here, we retrospect benign clinical and epidemiological outcomes-associated factors from a solved epidemic in a low epidemic area.

Methods: All 98 laboratory-confirmed COVID-19 patients in a local epidemic from January 17, 2020 to March 10, 2020 were enrolled. Data were updated until all patients having final outcomes. Patients were all hospitalized.

Results: The case fatality rate was $1.0 \%$. There were no local secondary infection cases. The severe/critical rate was $19.4 \%$. Compared with serious/critical cases, mild/ common cases on admission were much younger, lacks of comorbidities and normal in functions of vital organs and indicators of secondary bacterial infections. The lymphocyte counts in serious/critical cases began to be significantly lower 3 days before their identification dates. The absence of lymphopenia before the eighth day from disease onset can exclude the possibility of $78.5 \%$ to be serious/critical ill. Early antiviral treatment significantly shortened the viral RNA-negative conversion time, and its delay was associated with critical patients.

Conclusion: Early hospitalization of all patients to conduct antiviral treatment and to prevent secondary epidemic was the important benign clinical and epidemiological outcomes-associated factor of COVID-19. The active intervention strategies are crucial in low epidemic areas and the continuous monitoring of lymphocytes may be useful to sort patients reasonably in high epidemic areas.
\end{abstract}

\section{Introduction}

Coronavirus disease-2019 (COVID-19) has been worldwide confirmed in more than 99.9 million individuals with case fatality rate of $3.0 \%$ [1]. The severe patients of COVID-19 usually require costly intensive care and some of them evolve into adult respiratory distress syndrome with high mortality ignoring mechanical ventilation or extracorporeal membrane oxygenation [2].

The clinical features of COVID-19 have well documented in the literature [3-5], which have helped us understand the epidemiological, clinical, laboratory and radiological characteristics of this disease. Some studies have paid attentions to the mortality predictors [6-10]. The age of more than 65 years, pre-existing underlying diseases, the presence of secondary bacterial infections and the rises in blood inflammatory indicators are predictors of fatal outcomes. In addition, patients with severe infection are more likely to have diabetes, renal disease, and chronic pulmonary disease and have higher white blood cell counts, lower lymphocyte counts, and increased C-reactive protein $[11,12]$. The intensive care unit admission is predicted by raises in leukocyte count, alanine aminotransferase, aspartate transaminase, lactate dehydrogenase and procalcitonin [13]. However, these predictors are mainly summarized from high epidemic areas, usually from partial epidemic datasets in the early stage. The proposed models are indeed thought to be poorly reported, at high risk of bias, and their reported performance is probably optimistic [6].

The case fatality rate of COVID-19 significantly varies as the local intensity of epidemic. It is $6.6 \%$ in a high epidemic province, but only $0.8 \%$ in the rest provinces in China (updated on January 25, 2020), suggesting that there may be significant differences in mortality and disease severity predictors between high and low epidemic areas. On the other hand, a solved or complete epidemic may provide novel clues to reduce the deaths and secondary epidemic in high or new epidemic areas. For these reasons, we further investigated the benign clinical and epidemiological outcomes-associated factors of COVID-19 from a solved epidemic of 98 patients with one death and no local secondary infection cases.

${ }^{\star}$ Correspondence to: Xiao Mou Peng, Center of Infectious Diseases, the Fifth Affiliated Hospital, Sun Yat-sen University, 52 East Meihua Road, Zhuhai, Guangdong Province 519000, China, Tel: +86-756-2528500; E-mail: xiaomoupeng@hotmail.com

Key words: Coronavirus disease-19(COVID-19), clinical outcome, epidemiological outcome, clinical characteristics, antiviral treatment

Received: January 24, 2021; Accepted: January 28, 2021; Published: February 01, 2021 


\section{Methods}

\section{Study design and patients}

This single-center retrospective observational study was conducted in the Fifth Affiliated Hospital of Sun Yat-sen University, a designated hospital for COVID-19 in Zhuhai, Guangdong province, China. All 98 laboratory-confirmed COVID-19 patients in the local epidemic were hospitalized from 17 January 2020 to 10 March 2020.

\section{Data collection}

The epidemiological, demographical and clinical data, laboratory examination results, imaging reports and treatment regimens of all patients were extracted from the electronic medical records. The main symptoms, laboratory results and chest computed tomography (CT) findings on admission are presented and the final clinical classification was conducted based on entire disease course unless mentioned elsewhere.

\section{Laboratory confirmation}

Laboratory confirmation of severe acute respiratory syndrome coronavirus 2 (SARS-CoV-2) infection was performed at the Chinese Center for Disease Prevention and Control. SARS-CoV2 RNA in the nasal and pharyngeal swab specimens was detected using real-time RTPCR assays [4].

\section{Definitions}

According to guideline of diagnosis and treatment of COVID-19 issued by National Health Commission of China [14], those patients were clinically classified into mild, common, serious and critical types (Criteria are presented in supplementary materials).

\section{Treatments}

Most adult patients received antiviral treatment with lopinavir and ritonavir ( $400 \mathrm{mg}$ twice daily and $100 \mathrm{mg}$ twice daily for 14 days, respectively), arbidol (200 $\mathrm{mg}$ three times daily for 7-14 days) and/or chloroquine (500 mg once or twice daily for 7 days). Severe patients or patients with more than $50 \%$ progression in pneumonia within 48 hours were treated with corticosteroid (40-80 $\mathrm{mg}$ /day) and gamma globulin (15-20 g/day) for 3-5 days. Quinolones, macrolides or antifungal drugs were administered if there were co-infections with bacteria or fungus.

\section{Statistical analysis}

When the data were normally distributed, the continuous variables were expressed as mean \pm standard deviation (SD) and compared using student's t test and Pearson correlation analysis. When the data were non-normally distributed, the variables were expressed as median (interquartile range, IQR) and compared using Mann-Whitney U test and Spearman correlation analysis. The classification variables were expressed as number (\%) and compared by $\chi^{2}$ test or Fisher's exact test. $P<0.05$ was considered statistically significant. All data were analyzed using SPSS (version 23.0) software.

\section{Results}

\section{Clinical and epidemiological outcomes}

All 98 laboratory-confirmed COVID-19 patients in this local epidemic were hospitalized and included in our analysis. Data were continuously updated until all patients having final clinical outcomes (recovery or death). The epidemic had a benign epidemiological outcome without local secondary infection cases in health workers and local citizens. These patients, including 5 children (under 14 years old) and 15 elderly persons (over 65 years old), had benign clinical outcomes with a case fatality rate of $1.0 \%$ (1/98) (Table S1). Out of 98 cases, $13(13.3 \%)$ and $6(6.1 \%)$ belonged to serious and critical types, respectively. The serious/critical rate was $19.4 \%$ (19/98). All 6 (6.1\%) critical patients were admitted to the intensive care unit and $3(3.1 \%)$ of them underwent mechanical ventilation. Only a 78-years-old man with hypertension, coronary heart disease and diabetes died of adult respiratory distress syndrome. The mean period from disease onset to admission was 4.4 days. The average length of hospitalization was 21.1 days. The mean period of the first viral RNA negative conversion was 8.2 days. The rate of re-positive viral RNA was $18.4 \%$.

\section{Benign outcomes-associated factors in demographic and clinical characteristics}

The demographic and epidemiological characteristics of 98 COVID-19 patients were shown in Table S1. Mean age was 46.3 years and $45(45.9 \%)$ were male. When patients were stratified by the disease severity, the mean age of mild/common cases (43.2 years) was significantly younger than that of serious/critical cases (59.3 years) $(P<$ 0.001 ) (Table S1), suggesting that the younger age was a benign clinical outcome-associated factor. To confirm it, the mean age in 4 different clinical types and the occurrence of serious/critical cases in different age groups were compared (Figure 1). The less severity the cases, the younger the mean of age was (Figure 1A). The younger the group, the lower the proportion of patients with severe illnesses was (Figure 1B). The freeness of comorbidities seemed to be another benign clinical outcome-associated factor (Table S1). It was obvious, however, that the disease severity was associated with aging-related diseases, such as hypertension and diabetes. Only 3 cases had a history of respiratory disease, 2 of them had pulmonary tuberculosis. Fever, cough and sore throat were the three most common symptoms, accounting for $57.1 \%$, $39.8 \%$ and $9.2 \%$ respectively (Table $\mathrm{S} 1$ ).

\section{Benign outcomes-associated factors in Laboratory findings}

Laboratory and chest CT scan findings on admission were shown in Table S2. Blood routine examination showed that the decreases in eosinophil, lymphocyte and platelet counts were significantly associated with the serious/critical patients. The lymphopenia $(<$ $1.0 \times 10^{9} / \mathrm{L}$ ) occurred more frequently in serious/critical cases (Figure $2 \mathrm{~A})$. Furthermore, the counts of lymphocyte (Figure $2 \mathrm{~B}$ ), cluster of differentiation (CD) $3+$ (Figure 2C), CD3+CD4+ (Figure 2D) and $\mathrm{CD} 3+\mathrm{CD} 8+$ (Figure 2E) $\mathrm{T}$ lymphocytes and eosinophil (Figure 2F) significantly decreased in serious/critical patients. Moreover, when
A

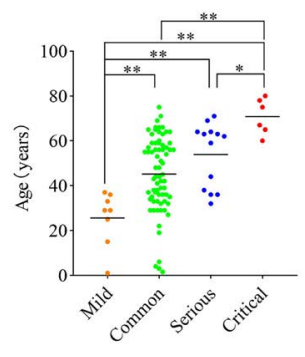

B

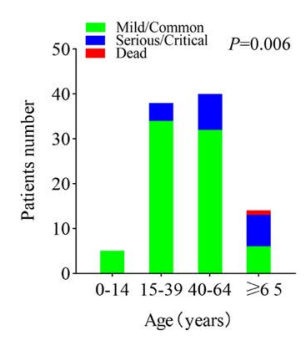

Figure 1. The mean age of different clinical types and the age distribution of COVID-19 patients. (A) Mean age of different clinical types of COVID-19 patients. ${ }^{*} P<0.05$, $* * P<0.01$. (B) Age distribution of COVID-19 patients 
A

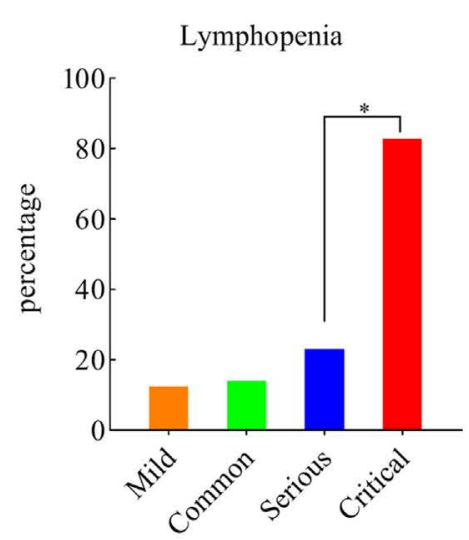

D

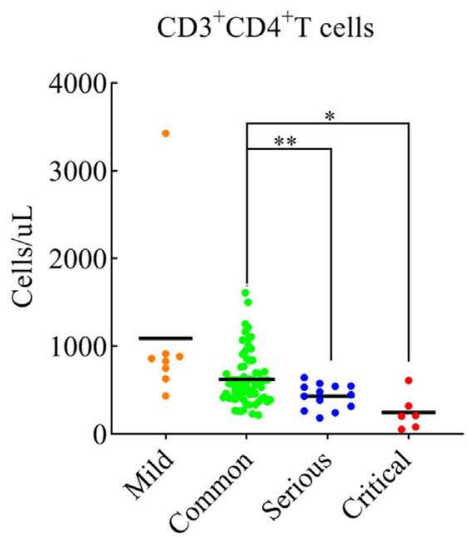

B

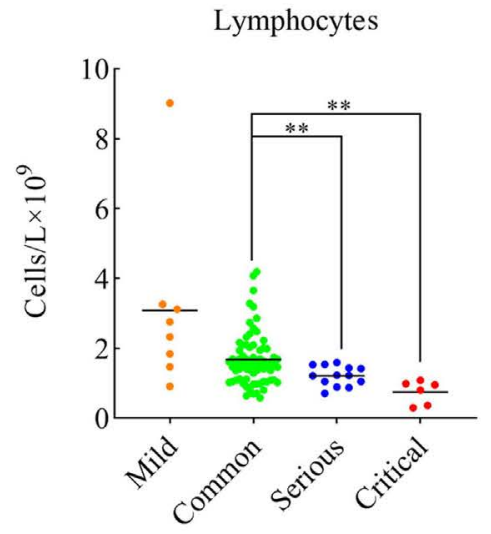

$\mathbf{E}$

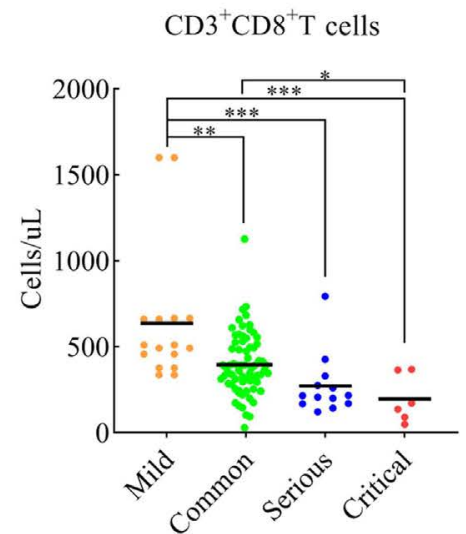

$\mathrm{CD}^{+}{ }^{+} \mathrm{T}$ cells

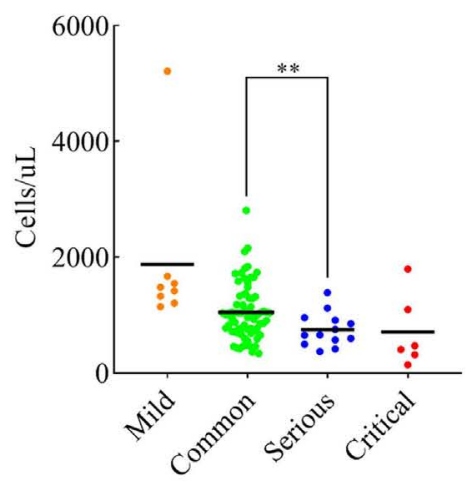

$\mathbf{F}$

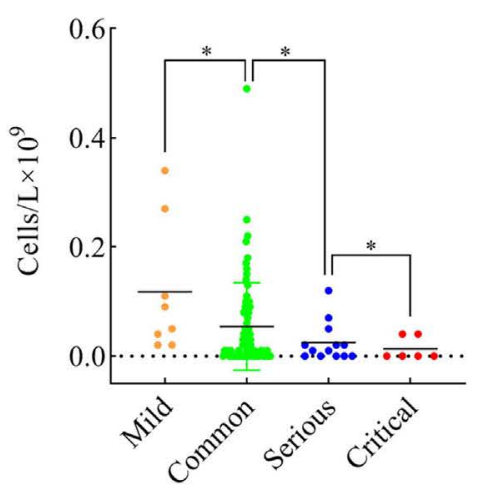

Figure 2. The lymphocyte and eosinophil counts and lymphocyte subsets in peripheral blood from different types of COVID-19 patients. (A) The occurrence of lymphopenia, (B) Lymphocyte counts, (C) CD3+T lymphocyte counts, (D) CD3+CD4+T lymphocyte counts, (E) CD3+CD8+T lymphocyte counts, and (F) eosinophil counts in different clinical types of COVID-19 patients. $* P<0.05, * * P<0.01$

compared the data before the identification date (an average of 7.8 days from disease onset) in serious/critical patients and the reference date (the eighth day) in mild/common cases, we found that the lymphocyte counts in serious/critical cases began to be significantly lower 3 days before their identification dates (Figure 3). As for mild/ common patients, the predictive sensitivity, predictive specificity, positive predictive value and negative predictive value of the absence of lymphopenia before the eighth day from disease onset were $78.5 \%$ (62/79), 89.5\% (17/19), 96.9\% (62/64) and 50.0\% (17/34), respectively. Simultaneously, bacterial infection indicators, C-reactive protein and procalcitonin, were elevated in a portion of serious/critical patients. Moreover, the decreases in serum albumin and increases in serum $\gamma$-globulin, total bilirubin, lactate dehydrogenase and D-dimer were significantly associated with the disease severity (Table S2), suggesting that admission of all patients before the occurrences of obvious dyspnea, substantial changes of vital organs and secondary bacterial infections were the major benign outcome-associated factors. Due to early admission, lung-related structure and function indicators, partial pressure of oxygen and chest CT scan except for partial pressure of carbon dioxide, did not changes more obviously in serious/critical cases on admission.

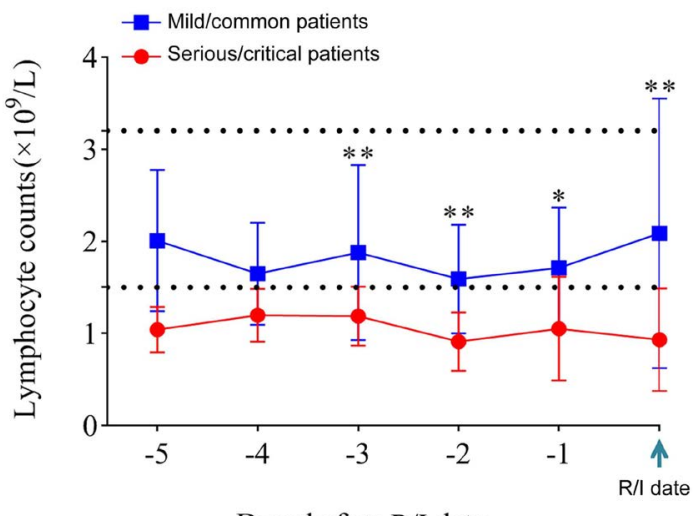

Days before R/I date

Figure 3. Early kinetic differences of lymphocyte counts in mild/common and serious critical patients of COVID-19. R/I date, reference date in mild/common patients/ identification date in serious/critical patients. The reference date was set as the eighth day that was equivalent to the mean time ( 7.8 days) for those serious/critical patients to be identified from disease onset. Lymphocyte counts of mild/common (blue line) and serious/ critical (red line) COVID-19 patients were analyzed at different time points before R/I date, respectively. Error bars, mean $\pm \mathrm{SD}$. ${ }^{*} P<0.05,{ }^{*} P<0.01$. The upper dotted lines show the upper normal limit and the lower dotted lines show the lower normal limit of lymphocyte counts 


\section{Benign outcomes-associated factors in treatments}

The general data about treatments of antivirals, antibiotics and corticosteroids were shown in Table S1. Chloroquine, arbidol and lopinavir/ritonavir were the major antivirals used in this local epidemic. Some patients used two or three antivirals simultaneously or sequentially. Among the different clinical types, the rates of antiviral treatment were similar (Figure 4A), but the average delayed time of antiviral treatment in critical cases was significantly longer than that in serious cases (Figure 4B), which was in concordance with the longer time for viral RNA-negative conversion (Figure 4C). The influences of antiviral treatment on clinical outcome indicators were shown in Table 1. The antiviral treatment started within 3 days from disease onset significantly shortened the time of viral RNA-negative conversion. Although it was not statistically significant, the rate of re-positive viral

\section{A}

\section{$\begin{array}{llll}87.5 & 88.9 & 92.3 & 83.3\end{array}$}

B

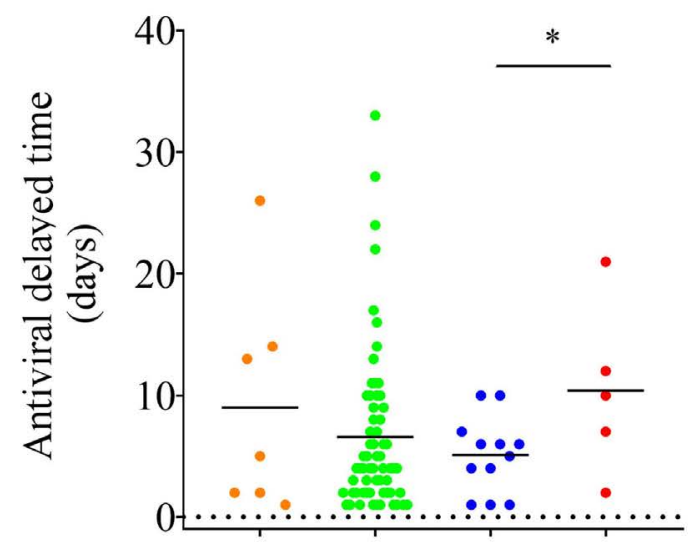

\section{C}

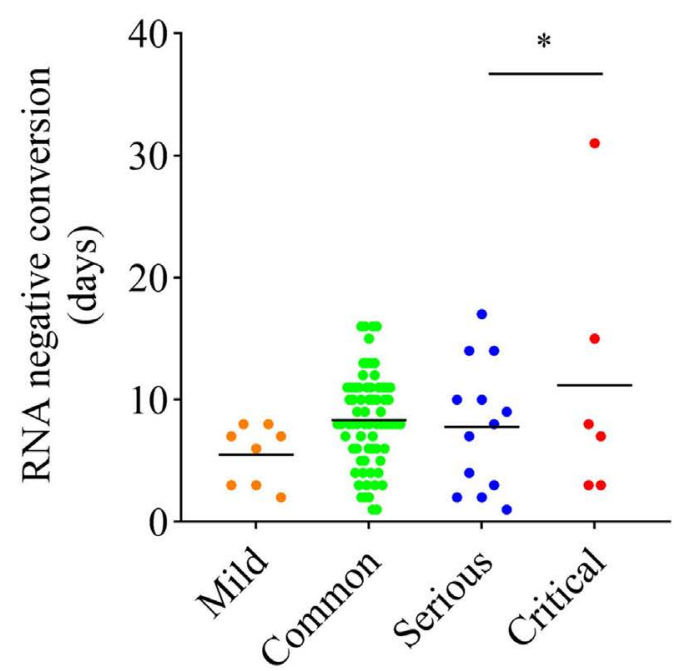

Figure 4. Antiviral therapy and time of SARS-Cov-2 RNA-negative conversion. (A) Rates of antiviral treatment, (B) Delayed time of antiviral therapy, and (C) Time of SARS-Cov-2 RNA-negative conversion in different clinical types of COVID-19 patients. $* P<0.05$
Table 1. Influences of treatments on the clinical outcomes

\begin{tabular}{|c|c|c|c|c|}
\hline & $\begin{array}{c}\text { Serous/critical } \\
\text { rate } \\
(\%)\end{array}$ & $\begin{array}{c}\text { RNA (-) } \\
\text { conversion } \\
\text { (days) }\end{array}$ & $\begin{array}{c}\text { RNA re- }(+) \\
\text { rate } \\
(\%)\end{array}$ & $\begin{array}{c}\text { Hospitalization } \\
\text { (days) }\end{array}$ \\
\hline \multicolumn{5}{|l|}{ Antivirals } \\
\hline$+(87)$ & $17(19.5 \%)$ & $8.41 \pm 0.50$ & $15(17.2 \%)$ & $21.6 \pm 0.87 *$ \\
\hline - (11) & $2(18.2 \%)$ & $6.55 \pm 0.95$ & $3(27.3 \%)$ & $17.3 \pm 2.16$ \\
\hline$\leq 3$ days (32) & $4(12.5 \%)$ & $7.06 \pm 0.81^{*}$ & $5(15.6 \%)$ & $21.3 \pm 1.39$ \\
\hline$>3$ days (35) & $13(23.6 \%)$ & $9.20 \pm 0.62$ & $10(18.2 \%)$ & $21.7 \pm 1.11$ \\
\hline \multicolumn{5}{|l|}{ Antibiotics } \\
\hline$+(71)$ & $18(25.4 \%)^{*}$ & $8.69 \pm 0.56^{*}$ & $10(14.1 \%)$ & $21.6 \pm 0.98$ \\
\hline - (27) & $1(3.70 \%)$ & $6.93 \pm 0.75$ & $8(29.6 \%)$ & $19.8 \pm 1.43$ \\
\hline$\leq 3$ days (30) & $6(20.0 \%)$ & $8.10 \pm 0.71$ & $6(20.0 \%)$ & $22.4 \pm 1.49$ \\
\hline$>3$ days (41) & $12(29.3 \%)$ & $9.12 \pm 0.83$ & $4(9.80 \%)$ & $21.0 \pm 1.31$ \\
\hline \multicolumn{5}{|c|}{ Corticosteroids } \\
\hline$+(15)$ & $10(66.7 \%)^{* *}$ & $9.67 \pm 1.84$ & $2(13.3 \%)$ & $20.5 \pm 1.64$ \\
\hline$-(83)$ & $9(10.8 \%)$ & $7.94 \pm 0.43$ & $16(19.7 \%)$ & $21.2 \pm 0.92$ \\
\hline
\end{tabular}

Data are shown in $\mathrm{n}(\%)$ or mean \pm standard deviation; Severe case, serious/critical types of patients; ${ }^{*} P<0.05,{ }^{*} P<0.01$, compared with the datum in the next line.

RNA was relatively lower in those patients who received antivirals. However, the hospitalization time was significantly longer in antiviralstreated patients, probably because there was a tendency to give antivirals to the relatively severe patients in each clinical type. Similar tendencies explained that antibiotics and corticosteroids elongated the time of viral RNA-negative conversion or were associated with a higher serious/critical rate. Thus, antiviral treatment was ascertained to be an important benign clinical outcome-associated factor. Simultaneously, the treatments of antibiotics and corticosteroids might be also contributable to the benign clinical outcome of serious/critical cases due to the low case fatality rate $(1.0 \%)$ in spite of the higher serious/ critical rate (19.4\%).

\section{Discussion}

Though COVID-19 evolved into a pandemic, there is a lack of standardized and convincing general solutions to stop the pandemic or for clinicians to reduce the deaths in clinical practice. It may be helpful to find some effective solutions by careful retrospection of solved epidemics. Zhuhai, China is a low epidemic area. The local epidemic began in early January and disappeared with a final case fatality rate of $1.0 \%$ and no local secondary infection cases at the end of March, 2020. Therefore, this local epidemic was suitable for the analysis of benign clinical and epidemiological outcomes-associated factors. By careful retrospection, we found that younger age, lack of aging-related diseases, admission of all patients before the occurrences of obvious dyspnea, substantial changes of vital organs, and secondary bacterial infections and early antiviral treatment were the major benign clinical and epidemiological outcomes-associated factors. Among these associated factors, early hospitalization seemed to be the most important one. It provided chances to conduct early antiviral treatment, antibacterial and antifungal treatments in times, reasonable care and anxiety release.

The clinical outcomes-associated factors of COVID-19 based on death cases have been broadly studied [6-10]. The fatal outcomes are correlated with the age of more than 65 years, pre-existing underlying diseases, the presence of secondary infections and the rises in blood inflammatory indicators. The disease severity is correlated with underling diseases such as diabetes, renal disease, and chronic pulmonary disease and abnormality of higher white blood cell counts, lower lymphocyte counts, and increased C-reactive protein $[11,12]$. Based on a solved epidemic in this study, we also found that the age and underlying comorbidities were the major factors associated with the 
disease severity. Moreover, the underlying comorbidities were mainly aging-related diseases such as hypertension and diabetes. Thus, age seemed to be the key disease severity-associated factor. The mean age in our dataset was 46.3 years, which is much younger than that reported from China ( $47-55$ years), Italy (65 years), Germany (65 years) and United States of America (65.5 years) [15-17]. By careful retrospection of the reports from a high epidemic area in china, the mean age was 42 years between January 16 and January 29, 2020 and 50-55 years between January 20 and February 14, 2020 [18,19], suggesting that the shift to the elders is the important contributing factor for the high case fatality in high epidemic area. Therefore, it is ascertained that younger age is the benign clinical outcome-associated factor of the epidemic episode in Zhuhai, China.

The commonest laboratory change of COVID-19 is lymphopenia $[4,20,21]$. Compared with mild/common ones, the serious/critical patients had significantly lower lymphocyte counts in this study. The lymphocyte counts in serious/critical cases began to be significantly lower 3 days before their identification dates. The absence of lymphopenia before the eighth day from disease onset can exclude the possibility of $78.5 \%$ to be serious/critical ill. These findings are helpful to reduce the pressure of the medical system by sorting patients reasonably in high epidemic areas. Except for clinical types-sorting dyspnea, those serious/critical patients on admission are more likely to have abnormalities in other vital organ functions, coagulation and secondary bacterial infections [9,22-24]. These abnormalities indeed frequently occurred in this study. However, compared with reports from high epidemic and high case fatality areas $[4,18,25]$, the overall symptoms (fever and cough) and laboratory changes of our patients were mild on admission, especially in the lung functions. The average time of our dataset from disease onset to admission was 4.4 days, and to diagnosis was 7.8 days, implying that most serious/critical patients were diagnosed after admission. Indeed, there were only 2 patients including the dead case had dyspnea on admission. In contrast, the average time for admission was 7.0 days in high epidemic and high case fatality rate area [25]. Therefore, laboratory data told us that early admission of all patients was the important benign clinical outcomeassociated factor in this study.

Though antivirals are worldwide expected, there are no approved antiviral agents up to now. However, some drugs including chloroquine, arbidol, remdesivir and lopinavir/ritonavir have shown aspiring effects on reduction of deaths [26-30]. In this study, most patients (88.8\%) completed antiviral regimen without obvious side effects, and the early antiviral treatment started within 3 days from disease onset significantly shortened the time of viral RNA-negative conversion, which was in concordance with early antiviral treatment contributing to alleviate the severity and improve the prognosis of patients [27], and no benefit was observed with lopinavir/ritonavir treatment beyond standard care in serious/critical patients [28]. The average delayed time of antiviral treatment in critical cases was significantly longer than that of serious cases, suggesting that early antiviral treatment might play a role in preventing from the serious-to-critical progression, perhaps were correlated with the low case fatality rate $(1.0 \%)$ in spite of the higher serious/critical rate (19.4\%) in our dataset.

Since massive migration is considered to promote the early spread of COVID-19 in China and China's practices with isolation of patients and quarantine of persons with close contacts [31,32], the early admissions of all patients and widespread antiviral therapy might be contributable for the benign epidemiological outcome of this local epidemic episode. Early admissions and early/widespread antiviral therapy might be helpful for the prevention of community and health worker infections. Nonetheless, to control this pandemic of COVID-19, there are many uncertainties. Concordantly, it is uncertain whether our successful experience is effective in high epidemic areas since the major death-contributable factor there is the lack of enough facilities. However, the continuous monitoring of lymphocytes may be useful to reduce the pressure of the medical system by sorting patients reasonably. We also strongly suggest that the conducts of early isolation and general antiviral therapy to mild or young patients who are suited for most current potential antivirals are imperative to reduce the deaths by preventing old or fragile persons from infection in high epidemic areas.

\section{Funding}

This work was supported by COVID-19 special fund 2020 from Science and technology Bureau of Zhuhai, Guangdong Province, China. The funding source did not have a role in collection, analysis and interpretation of data.

\section{Conflict of interest}

The authors declare that they have no conflict of interest.

\section{Ethical approval}

This study was approved by the ethics committee of the Fifth Affiliated Hospital of Sun Yat-sen University.

\section{References}

1. Johns Hopkins University (2020) COVID-19 dashboard by the Center for Systems Science and Engineering (CSSE) at Johns Hopkins University (JHU).

2. Yang X, Yu Y, Xu J, Shu H, Xia J, et al. (2020) Clinical course and outcomes of critically ill patients with SARS-CoV-2 pneumonia in Wuhan, China: A single-centered, retrospective, observational study. Lancet Respir Med 8: 475-481.

3. Wei Y, Zeng W, Huang X, Li J, Qiu X, Li H, et al. (2020) Clinical characteristics of 276 hospitalized patients with coronavirus disease 2019 in Zengdu District, Hubei Province: a single-center descriptive study. BMC Infect Dis 20: 549. [Crossref]

4. Guan WJ, Ni ZY, Hu Y, Liang WH, Ou CQ, et al. (2020) Clinical Characteristics of Coronavirus Disease 2019 in China. N Engl J Med 382: 1708-1720.

5. Zhao XY, Xu XX, Yin HS, Hu QM, Xiong T, et al. (2020) Clinical characteristics of patients with 2019 coronavirus disease in a non-Wuhan area of Hubei Province, China: a retrospective study. BMC Infect Dis 20: 311. [Crossref]

6. Wynants L, Van Calster B, Collins GS, Riley RD, Heinze G, et al. (2020) Prediction models for diagnosis and prognosis of covid-19 infection: systematic review and critical appraisal. BMJ 69: m1328. [Crossref]

7. Tian W, Jiang W, Yao J, Nicholson CJ, Li RH, et al. (2020) Predictors of mortality in hospitalized COVID-19 patients: A systematic review and meta-analysis. J Med Virol 92: 1875-1883. [Crossref]

8. Ruan Q, Yang K, Wang W, Jiang L, Song J (2020) Clinical predictors of mortality due to COVID-19 based on an analysis of data of 150 patients from Wuhan, China. Intensive Care Med 46: 846-848. [Crossref]

9. Wang F, Nie J, Wang H, Zhao Q, Xiong Y, et al. (2020) Characteristics of periphera lymphocyte subset alteration in COVID-19 pneumonia. J Infect Dis 221: 1762-1769. [Crossref]

10. Du RH, Liang LR, Yang CQ, Wang W, Cao TZ, et al. (2020) Predictors of mortality for patients with COVID-19 pneumonia caused by SARS-CoV-2: a prospective cohort study. Eur Respir J 55: 2000524. [Crossref]

11. Wang D, Li R, Wang J, Jiang Q, Gao C, et al. (2020) Correlation analysis between disease severity and clinical and biochemical characteristics of 143 cases of COVID-19 in Wuhan, China: a descriptive study. BMC Infect Dis 20: 519. [Crossref]

12. Ji D, Zhang D, Xu J, Chen Z, Yang T, et al. (2020) Prediction for Progression Risk in Patients with COVID-19 Pneumonia: the CALL Score. Clin Infect Dis 71: 1393-1399. [Crossref] 
13. Foieni F, Sala G, Mognarelli JG, Suigo G, Zampini D, et al. (2020) Derivation and validation of the clinical prediction model for COVID-19. Intern Emerg Med 15: 14091414. [Crossref]

14. http://www.nhc.gov.cn/yzygi/s7653p/202003/46c9294a7dfe4cef80dc7f5912eb1989.shtml

15. Aggarwal S, Garcia-Telles N, Aggarwal G, Lavie C, Lippi G, et al. (2020) Clinical features, laboratory characteristics, and outcomes of patients hospitalized with coronavirus disease 2019 (COVID-19): Early report from the United States. Diagnosis (Berl) 7: 91-96. [Crossref]

16. Ciceri F, Castagna A, Rovere-Querini P, De Cobelli F, Ruggeri A, et al. (2020) Early predictors of clinical outcomes of COVID-19 outbreak in Milan, Italy. Clin Immunol 217: 108509. [Crossref]

17. Hoffmann C, Wolf E (2020) Older age groups and country-specific case fatality rates of COVID-19 in Europe, USA and Canada. Infection 24: 1-6. [Crossref]

18. Li R, Tian J, Yang F, Lv L, Yu J, et al. (2020) Clinical characteristics of 225 patients with COVID-19 in a tertiary Hospital near Wuhan, China. J Clin Virol 127:104363. [Crossref]

19. Zhang G, Hu C, Luo L, Fang F, Chen Y, et al. (2020) Clinical features and short-term outcomes of 221 patients with COVID-19 in Wuhan, China. J Clin Virol 127: 104364 [Crossref]

20. Liu J, Li S, Liu J, Liang B, Wang X, et al. (2020) Longitudinal characteristics of lymphocyte responses and cytokine profiles in the peripheral blood of SARS-CoV-2 infected patients. EbioMedicine 55: 102763. [Crossref]

21. Tan L, Wang Q, Zhang D, Ding J, Huang Q, et al. (2020) Lymphopenia predicts disease severity of COVID-19: a descriptive and predictive study. Signal Transduct Target Ther 5: 33. [Crossref]

22. Lippi G, Plebani M, Henry BM (2020) Thrombocytopenia is associated with severe coronavirus disease 2019 (COVID-19) infections: A meta-analysis. Clin Chim Acta 506: 145-148. [Crossref]
23. Wang F, Nie J, Wang H, Zhao Q, Xiong Y, et al. (2020) Characteristics of peripheral lymphocyte subset alteration in COVID-19 pneumonia. $J$ Infect Dis 221: 1762-1769. [Crossref]

24. Liu F, Xu A, Zhang Y, Xuan W, Yan T, et al. (2020) Patients of COVID-19 may benefit from sustained Lopinavir-combined regimen and the increase of Eosinophil may predict the outcome of COVID-19 progression. Int J Infect Dis 95: 183-191. [Crossref]

25. Chan KW, Wong VT, Tang SCW (2020) COVID-19: An Update on the Epidemiological, Clinical, Preventive and Therapeutic Evidence and Guidelines of Integrative ChineseWestern Medicine for the Management of 2019 Novel Coronavirus Disease. Am J Chin Med 48: 737-762. [Crossref]

26. Stebbing J, Phelan A, Griffin I, Tucker C, Oechsle O, et al. (2020) COVID-19 combining antiviral and anti-inflammatory treatments. Lancet Infect Dis 20: 400-402. [Crossref]

27. Wu J, Li W, Shi X, Chen Z, Jiang B, et al. (2020) Early antiviral treatment contributes to alleviate the severity and improve the prognosis of patients with novel coronavirus disease (COVID-19). J Intern Med 288: 128-138.

28. Cao B, Wang Y, Wen D, Liu W, Wang J, et al. (2020) A Trial of Lopinavir-Ritonavir in Adults Hospitalized with Severe Covid-19. N Engl J Med 382: 1787-1799. [Crossref]

29. Fanin A, Calegari J, Beverina A, Tiraboschi S, Gruppo di Autoformazione Metodologica (GrAM) (2020) Hydroxychloroquine and azithromycin as a treatment of COVID-19. Intern Emerg Med 15: 841-843. [Crossref]

30. Ferner RE, Aronson JK (2020) Remdesivir in covid-19. BMJ 369: m1610. [Crossref]

31. Song WY, Zang P, Ding ZX, Fang XY, Zhu LG, et al. (2020) Massive migration promotes the early spread of COVID-19 in China: a study based on a scale-free network. Infect Dis Poverty 9: 109.

32. Xu TL, Ao MY, Zhou X, Zhu WF, Nie HY, et al. (2020) China's practice to prevent and control COVID-19 in the context of large population movement. Infect Dis Poverty 9 115. [Crossref]

Copyright: (C2021 Gang Hu. This is an open-access article distributed under the terms of the Creative Commons Attribution License, which permits unrestricted use, distribution, and reproduction in any medium, provided the original author and source are credited. 